\title{
Fauna parasitária de peixes oriundos de "pesque-pagues" do município de Franca, São Paulo, Brasil. II. Metazoários
}

\author{
Marcos Tavares-Dias ${ }^{1}$ \\ Flávio R. Moraes ${ }^{1,2}$ \\ Maurício L. Martins ${ }^{1}$ \\ Sérgio N. Kronka ${ }^{3}$
}

\begin{abstract}
Parasitic fauna of cultivated fishes in feefishing farm of franca, state of São Paulo, Brazil. II. Metazoans. Metazoan fauna from cultivated físhes ( $N=433$ ) in two feefishing farm of Franca, State of São Paulo, Brazil, was studied during a period of April 1997 through March 1999. Specimens of piauçú Leporinus macrocephalus Garavello \& Britski, 1988 (Anostomidae), pacu Piaractus mesopotamicus Holmberg, 1887 (Characidae), carp Cyprinus carpio Linnaeus, 1758 (Cyprinidae), Tillapia rendalli Boulenger, 1896 (Cichlidae), nile-tilapia Oreochromis niloticus Linnaeus, 1758 (Cichlidae), matrinxã Brycon cephalus Günther, 1869 (Characidae) and tambacu hybrid (male of $P$. mesopotamicus $\mathrm{x}$ female of Colossoma macropomum Cuvier, 1818) were collected. A total of $29.3 \%$ was parasitized by monogenean, $9.7 \%$ with crustacean (copepodits and adults of Lernaea cyprinacea Linnaeus, 1758 and Argulus sp.) and $1.6 \%$ with myxosporean (Henneguya piaractus Martins \& Souza, 1997 and Myxobolus colossomatis Molnar \& Békési, 1993).The most susceptible species to myxosporean infestation was $P$. mesopotamicus. $L$. macrocephalus and $P$. mesopolanicus that showed a higher susceptibility to Argulus sp infestation. It was not observed significant difference $(\mathrm{P}>0.05)$ in the seasonal variation of $L$. cyprinacea infection of all species. L. macrocephalus, P. mesopotamicus and tambacu showed a great number of $P$. pillulare with no significant difference $(P>0.05)$ between them. Monogenean infections were observed in all examined species, without significant difference $(P>0.05)$ in the seasonal variation. Nevertheless, occurrence of monogenean was higher in P. mesopotamicus and lower in Nile-tilapia. By the other hand, parasites number collected from $P$. mesopotamicus was lower in the winter. Copepodits of $L$. cyprinacea were found in the gills of $P$. mesopotamicus, tambacu and $L$. macrocephalus. However, adults of $L$. cyprinacea were observed only in the $P$. mesopotanicus and tambacu.
\end{abstract}

KEY WORDS. Crustacean, copepodits, freshwater fish, metazoans, monogenean, myxosporeans, parasites, helminths

O ambiente aquático de criatórios artificiais facilita a invasão nos peixes por agentes patogênicos graças à maior concentração de animais por unidade de espaço, quando comparada à de ambiente natural. Além disso, a limitação imposta aos

1) Laboratório de Patologia Organismos Aquáticos, Centro de Aqüicultura (CAUNESP). Via de Acesso Prof. Paulo Donato Castellane, 14870-000 Jaboticabal, São Paulo, Brasil. E-mail: mtavares@caunesp.unesp.br

2) Departamento de Patologia Veterinária, FCAV, Universidade Estadual Paulista. Via de Acesso Prof. Paulo Donato Castellane, 14870-000 Jaboticabal, São Paulo, Brasil.

3) Departamento de Ciências Exatas, Universidade Estadual Paulista. Via de Acesso Prof. Paulo Donato Castellane, 14870-000 Jaboticabal, São Paulo, Brasil. Pesquisador do CNPq. 
predadores de peixes doentes também colabora para perpetuação e difusão dos patógenos no ambiente (ROBERTS 1981; RANZANI-PAIVA et al. 1989; THATCHER \& BRITES-NETO 1994).

Os parasitos são as maiores causas de perdas econômicas em peixes cultivados, sendo de maior relevância no neotrópico, pelas características climáticas pertinentes à região, que propiciam suas rápida e constante propagação (THATCHER \& BRITES-NETO 1994). A aqüicultura é responsabilizada pelo caráter cosmopolita de alguns parasitos como por exemplo, a Lernaea cyprinacea Linnaeus, 1758 (Crustacea) e o Argulus foliaceus Linnaeus, 1758 (DELARIVA \& AgostinHo 1999).

A piscicultura brasileira passa por uma fase de consolidação e expansão inquestionáveis (CHAMMAS 1997) e, nos últimos anos, ocorreu marcada expansão de empreendimentos conhecidos como "pesque-pague". Esses são utilizados para a pesca esportiva, atividade social e econômica de relevância nos países desenvolvidos (Grosman 1999). Apenas no Estado de São Paulo, calcula-se que existam mais de 3.000 "pesque-pague", nos quais podem ser encontradas inúmeras espécies de peixes (DIAS 1998). Embora ainda não avaliados, os "pesque-pague" devem se constituir em importantes riscos adicionais de disseminação de espécies exóticas de peixes e parasitos (ORSI \& AGOSTINHO 1999). Considerando que a maioria desses estabelecimentos não produz peixes, há necessidade de grande número de pisciculturas para abastecer esse mercado (EIRAS et al. 1998). Assim o transporte de animais é questão relevante do ponto de vista epidemiológico, uma vez que pode atuar como elemento disseminador de enfermidades (KENNEDY 1993; CHÁvEZ-MANCILLA 1985; Eiras et al. 1998; Delariva \& AGOSTINHO 1999).

Neste país, existem registros de diversos parasitos com potencial patogênico para os peixes destacando-se os metazoários mixosporídeos dos gêneros Myxobolus Butschi, 1882 e Henneguya Thilokan, 1892, os helmintos monogenóides e o crustáceo L. cyprinacea (CECCARELli et al. 1990; FIGUEIRA \& CECCARELLI 1991; BÉKÉSI 1992; MARTINS 1999 a,b).

Este trabalho teve como objetivo avaliar a ocorrência e a sazonalidade de parasitos metazoários em peixes cultivados em dois "pesque-pague" do município de Franca, Estado de São Paulo, no período compreendido entre abril de 1997 a março de 1999.

\section{MATERIAL E MÉTODOS}

No presente trabalho, empregou-se a mesma metodologia descrita em TAVARES-DiAs et al. (2001), com exceção dos monogenóides e copepoditos de L. cyprenacea, que após sedimentação, foram contados com auxílio de estereomicroscópio. Os crustáceos adultos foram contados a olho nú.

\section{RESULTADOS}

\section{Características da água}

Os valores médios da temperatura da água e o $\mathrm{pH}$, durante o período de abril de 1997 a março de 1999, assim como a condutividade elétrica e oxigênio dissolvido, no período de abril de 1998 a março de 1999, no "pesque-pague" $1 \mathrm{MG}$ e 2RB são aqueles mesmos demonstrados na figura 1A-B de TAVARES-DiAS et al. (2001). 


\section{Ocorrência e carga parasitária média}

No período de abril de 1997 a março de 1999, em ambos "pesque-pague" foram necropsiados 433 espécimes de teleósteos, sendo 111 piauçús Leporinus macrocephalus Garavello \& Britski, 1988 (medindo de 21,5 a 46,0 cm e pesando de 110,0 a 1360 g), 92 pacus Piaractus mesopotamicus Holmberg, 1887 (medindo de 15,0 a 47,0 cm e pesando de 80,0 a 2000,0 g), 09 carpas Cyprinus carpio Linnaeus, 1758 (medindo de 21,0 a 45,5 cm e pesando de 110,0 a $1500,0 \mathrm{~g}$ ), 09 Tillapia rendalli Boulenger, 1896 (medindo de 16,0 a $23,5 \mathrm{~cm}$ e pesando de 80,0 a 250,0 g), 110 tilápias-do-Nilo Oreochromis niloticus Linnaeus, 1757 (medindo de 20,0 a 34,5 cm e pesando de 160,0 a 750,0 g), 26 matrinxãs Brycon cephalus Günther, 1869 (medindo de 33,5 a 48,0 cm e pesando de 610,0 a 1980,0 g) e 76 tambacus (macho de P. mesopotamicus x fêmea de Colossoma macropomum Cuvier, 1818 (medindo de 17,0 a 50,0 cm e pesando de 100,0 a 2270,0 g).

A análise parasitológica dos fragmentos de rim, fígado, baço e coração não revelou a presença de parasitos ou alterações dignas de menção.

A carga parasitária média e a ocorrência de parasitos metazoários em peixes necropsiados $(\mathrm{N}=226)$ no "pesque-pague" $1 \mathrm{MG}$, no período de abril de 1997 a março de 1999, estão discriminadas na tabela I. Somente as brânquias de $P$. mesopotamicus estavam parasitadas por mixosporídeos. Esses foram identificados como Henneguya piaractus Martins \& Souza, 1997 (Myxobolidae) e Myxobolus colossomatis Molnar \& Békési, 1993 (Myxobolidae). Infecção por helmintos monogenóides ocorreu em $P$. mesopotamicus, L. macrocephalus, tambacu, $O$. niloticus e B. cephalus. Entretanto, o P. mesopotamicus foi o hospedeiro com maior ocorrência e abundância média do Monogenoidea Anacanthorus penilabiatus Böeger, Husack \& Martins, 1995 (Dactylogyridae), ao contrário da tilápia-do-Nilo que foi menos susceptível a monogenéticos. Espécimes adultos de L. cyprinacea foram encontrados somente na superfície corporal de tambacus. Porém, copepoditos desse crustáceo foram observados nas brânquias de pacus, tambacus e piauçús. Já o crustáceo Argulus sp. foi observado na superfície corporal de pacus e piuçús.

No "pesque-pague" $2 \mathrm{RB}$, foram examinados 207 peixes e a maior ocorrência de infecção por monogenea ocorreu em $P$. mesopotamicus e L. macrocephalus, quando comparado ao tambacu, $T$. rendalli, C. carpio e O. niloticus. Porém, monogenea (A. penilabiatus) foi observado em maior quantidade nas brânquias de $P$. mesopotamicus. Somente $P$. mesopotamicus foram infestados por adultos de $L$. cyprinacea. Mas, copepoditos de $L$. cyprinacea foram encontrados nas brânquias de P. mesopotamicus e L. macrocephalus. Enquanto as infecções por mixosporídeos H. piaractus ocorreram somente em P. mesopotamicus (Tab. II).

Os resultados demonstraram que nos dois "pesque-pague" $29,3 \%$ dos hospedeiros estavam infectados por helmintos Monogenea; $9,7 \%$ por crustáceos e 1,6\% por mixosporídeos (Fig. 1). Todos os parasitos foram encontrados nas brânquias dos peixes, exceto adultos de L. cyprinacea e Argulus sp., que infestavam a superfície corporal dos peixes.

Das espécies necropsiadas nos dois "pesque-pague", somente P. mesopotamicus foram infectados com mixosporídeos ( $M$. colossomatis e H. piaractus), 
Tabela I. Carga parasitária média e ocorrência (\%) de parasitos metazoários em 226 peixes necropsiados no "pesque-pague" 1 MG, período de abril de 1997 a março de 1999, no município de Franca, São Paulo. (N) Número de peixes infectados.

\begin{tabular}{|c|c|c|c|c|c|c|c|c|c|c|}
\hline \multirow{2}{*}{$\begin{array}{l}\text { Hospedeiros } \\
\text { Parasitos }\end{array}$} & \multicolumn{2}{|c|}{ P. mesopotamicus $(\mathrm{N}=44)$} & \multicolumn{2}{|c|}{ L. macrocephalus $(\mathrm{N}=29)$} & \multicolumn{2}{|c|}{ Tambacu $(\mathrm{N}=20)$} & \multicolumn{2}{|c|}{ B. cephalus $(\mathrm{N}=5)$} & \multicolumn{2}{|c|}{ O. niloticus ( $N=7$ ) } \\
\hline & Carga parasitária & $\%$ & Carga parasitária & $\%$ & Carga parasitária & $\%$ & Carga parasitária & $\%$ & Carga parasitária & $\%$ \\
\hline Mixosporideos & - & 7.0 & - & - & - & - & - & - & - & - \\
\hline Monogenea & $920,8 \pm 1841,0$ & 71.0 & $190,0 \pm 146,0$ & 40,0 & $293,5 \pm 233,0$ & 13,0 & $240,4 \pm 57,3$ & 18,2 & $31,4 \pm 20,4$ & 12,8 \\
\hline L. cyprinacea & - & - & - & - & $5,8 \pm \quad 4,9$ & 28,0 & - & - & - & - \\
\hline Copepoditos* & $9,0 \pm 11,6$ & 6,0 & $12,0 \pm 11,3$ & 7,0 & $8,7 \pm 5,4$ & 9,0 & - & - & - & - \\
\hline Argulus sp. & $6,0 \pm \quad 2,5$ & 2,0 & $5,0 \pm \quad 2,0$ & 2,0 & - & - & - & - & - & - \\
\hline
\end{tabular}

*) Copepoditos de L. cyprinacea; (N) número de peixes infectados.

Tabela II. Carga parasitária e ocorrência (\%) de parasitos metazoários em 207 peixes necropsiados no "pesque-pague" 2RB, período de abril de 1997 a março de 1999, no municipio de Franca, São Paulo.

\begin{tabular}{|c|c|c|c|c|c|c|c|c|c|c|c|c|c|}
\hline \multirow{2}{*}{$\begin{array}{c}\text { Hospedeiros } \\
\text { Parasitos }\end{array}$} & \multicolumn{3}{|c|}{ P. mesopotamicus $(\mathrm{N}=29)$} & \multicolumn{2}{|c|}{ L. macrocephalus $(\mathrm{N}=49)$} & \multicolumn{2}{|c|}{ Tambacu $(\mathrm{N}=20)$} & \multicolumn{2}{|c|}{ T. rendalli $(\mathrm{N}=1)$} & \multicolumn{2}{|c|}{ O. niloticus $(\mathrm{N}=3$ ) } & \multicolumn{2}{|c|}{ C. carpio $(n=4)$} \\
\hline & Carga pa & itária & $\%$ & Carga parasitária & $\%$ & Carga parasitária & $\%$ & Carga parasitária & $\%$ & Carga parasitária & $\%$ & Carga parasitária & $\%$ \\
\hline Mixosporídeos & - & & 6,0 & - & - & - & - & - & - & - & - & - & - \\
\hline Monogenea & $4600,7 \pm$ & 327,4 & 87,0 & $285,2 \pm 344,6$ & 79,0 & $644,2 \pm 951,7$ & 10,0 & $29,0 \pm 0,0$ & 14,3 & $31,0 \pm 0,0$ & 1,0 & $31,0 \pm 43,3$ & 37,0 \\
\hline L. cyprinacea & $2,0 \pm$ & 0,0 & 2,1 & - & - & - & - & - & - & - & - & - & - \\
\hline Copepoditos ${ }^{\star}$ & $10,3 \pm$ & 4,2 & 10,0 & $6,7 \pm$ & 15,0 & - & - & - & - & - & - & - & - \\
\hline
\end{tabular}

*) Copepoditos de L. cyprinacea; (N) número de peixes infectados.

Tabela III. Carga parasitária média e ocorrência (\%) de parasitos metazoários em 433 peixes necropsiados no período de abril de 1997 a março de 1999, em "pesque-pagues" do município de Franca, São Paulo.

\begin{tabular}{|c|c|c|c|c|c|c|c|c|c|c|c|c|c|c|c|c|c|}
\hline \multirow{2}{*}{$\begin{array}{c}\text { Hospedeiros } \\
\text { Parasitos }\end{array}$} & \multicolumn{3}{|c|}{$\begin{array}{l}\text { P. mesopotamicus } \\
(\mathrm{N}=73)\end{array}$} & \multicolumn{3}{|c|}{$\begin{array}{l}\text { L. macrocephalus } \\
\qquad(\mathrm{N}=78)\end{array}$} & \multicolumn{3}{|c|}{$\begin{array}{l}\text { Tambacu } \\
(\mathrm{N}=37)\end{array}$} & \multicolumn{2}{|l|}{$\begin{array}{l}\text { B. cephalus } \\
(\mathrm{N}=7)\end{array}$} & \multicolumn{2}{|l|}{$\begin{array}{l}\text { T. rendalli } \\
(\mathrm{N}=2)\end{array}$} & \multicolumn{2}{|l|}{$\begin{array}{l}\text { O. niloticus } \\
(\mathrm{N}=10)\end{array}$} & \multicolumn{2}{|l|}{$\begin{array}{l}\text { C. carpio } \\
(n=4)\end{array}$} \\
\hline & Carga par & sitária & $\%$ & Carga par & sitária & $\%$ & Carga par & sitária & $\%$ & Carga parasitária & $\%$ & Carga parasitária & $\%$ & Carga parasitária & $\%$ & Carga parasitária & $\%$ \\
\hline Mixosporideos & - & & 6,0 & - & & - & - & & - & - & - & - & - & - & - & - & - \\
\hline Monogenea & $2287,6 \pm$ & 6630,4 & 76,0 & $251,5 \pm$ & 292,4 & 58,0 & $433,8 \pm$ & 6040,0 & 11,7 & $1720,0 \pm 125,4$ & 18,7 & $24,5 \pm 4,5$ & 22,2 & $25,5 \pm 7,8$ & 5,4 & $31,0 \pm 43,3$ & 33,0 \\
\hline Copepoditos* & $9,6 \pm$ & 8,6 & 7,0 & $8,5 \pm$ & 7,8 & 11,0 & $13,4 \pm$ & 11,4 & 5,9 & - & - & - & - & - & - & - & - \\
\hline L. cyprinacea & $2,0 \pm$ & 0,0 & 2,1 & - & & - & $2,6 \pm$ & 2,4 & 15,3 & - & - & - & - & - & - & - & - \\
\hline Argulus sp. & $5,0 \pm$ & 2,0 & 1,0 & $4,0 \pm$ & 3,0 & 0,9 & - & & - & - & - & - & - & - & - & - & - \\
\hline
\end{tabular}


enquanto todas as outras espécies albergavam monogenóides, especialmente o $L$. macrocephalus $(76,0 \%)$, o P. mesopotamicus $(58,0 \%)$, C. Carpio $(33,3 \%)$ e a $T$. rendalli $(22,2 \%)$. As maiores ocorrências e carga média do Monogenea $A$. penilabiatus ocorreram nos espécimes de $P$. mesopotamicus enquanto a tilápiado-Nilo $(5,7 \%)$ apresentava baixos níveis de parasitismo por outros helmintos monogenóides (Tab. III).

Copepoditos de L. cyprinacea foram encontradas nas brânquias de 7,0\% dos P. mesopotamicus necropsiados, em 5,9\% dos tambacus e em $11,0 \%$ dos $L$. macrocephalus. Já, as formas adultas de $L$. cyprinacea foram observadas apenas em P. mesopotamicus $(2,1 \%)$ e tambacus $(15,3 \%)$, sendo esse último hospedeiro mais susceptível à infestação. A infestação por Argulus sp. foi baixa, e ocorreu somente em $0,9 \%$ da superfície corporal dos L. macrocephalus e em 1,0\% dos P. mesopotamicus examinados (Tab. III).

A associação entre parasitos metazoários e protozoários foi mais freqüente em P. mesopotamicus e L. macrocephalus, que em tambacus. Em P. mesopotamicus e $L$. macrocephalus houve associação entre Monogenea e $P$. pillulare; Monogenea e Trichodina sp.; Monogenea e I. multifilis e entre Monogenea e copepoditos de L. cyprinacea (Tab. IV).

Tabela IV. Percentual de ocorrência das principais associações parasitárias nas brânquias de peixes examinados no período de abril de 1997 a março de 1999, em "pesque-pagues" do município de Franca, São Paulo.

\begin{tabular}{lccc}
\hline \multicolumn{1}{c}{ Associações } & Tambacu & P. mesopotamicus & L. macrocephalus \\
\hline Monogenea / P. pillulare & 3,5 & 20,6 & 26,1 \\
Monogenea / Trichodina sp. & - & 10,9 & 9,0 \\
Monogenea / I. multifilis & - & 8,7 & 3,6 \\
Monogenea / Copepoditos & - & 3,3 & 7,2 \\
\hline
\end{tabular}

(*) Copepoditos de L. cyprinacea.

\section{Variação sazonal da ocorrência de parasitos}

Em P. mesopotamicus, O. niloticus, tambacu e L. macrocephalus a ocorrência de monogenea não sofreu influência sazonal. Entretanto, no tambacu não houve infecção nos meses de inverno e verão (Fig. 2A). Porém, carga média de monogenea em P. mesopotamicus e $L$. macrocephalus foi menor nos meses de inverno. A infecção por Monogenea (A. penilabiatus) em P. mesopotamicus, aumentou juntamente com temperatura, nos meses de primavera e verão (Fig. 2B).

A ocorrência de copepoditos de L. cyprinacea em L. macrocephalus não evidenciou diferença significativa $(P>0,05)$ entre as estações de outono, inverno, primavera e verão. Entretanto, não houve infestação por copepoditos em L. macrocephalus e tambacu, nos meses de inverno, enquanto em $P$. mesopotamicus, a ocorrência dessas formas imaturas atingiu o máximo na primavera (Fig. 3A). O número de copepoditos nesses três hospedeiros decresceu dos meses de outono para os de inverno, voltando a aumentar na primavera. Porém, em P. mesopotamicus e tambacu houve declínio no número de copepoditos da primavera para o verão, ao contrário do que ocorreu em L. macrocephalus (Fig. 3B). 


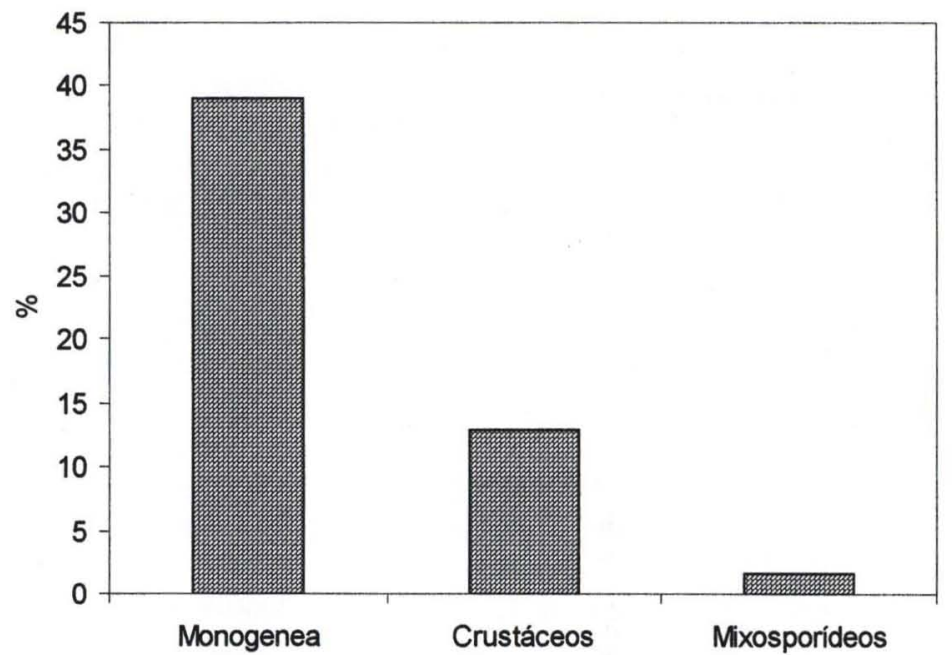

Fig. 1. Ocorrência dos grupos de metazoários em 211 hospedeiros parasitados, no periodo de abril de 1997 a março de 1999, em "pesque-pagues" do município de Franca, São Paulo.

Em L. macrocephalus e tambacu não houve diferença significativa $(\mathrm{P}>0,05)$ na ocorrência de L. cyprinacea entre as estações do ano. Porém, no tambacu, a maior carga média foi observada na primavera e verão, período de maior temperatura. Já em $P$. mesopotamicus a infestação ocorreu somente no outono, enquanto em $L$. macrocephalus a presença de L. cyprinacea ocorreu na primavera e verão, sendo maior o número parasitos na primavera (Fig. 4A-B).

\section{DISCUSSÃO}

O surgimento de enfermidades em dada população é um fenômeno dinâmico, pois as variações na ocorrência e no impacto sobre os peixes são dependentes das interações entre hospedeiro, patógeno e ambiente (RENO 1998; HEDRICK 1998).

Em ambos os "pesque-pague" a ocorrência de parasitismo foi similar. Os parasitos metazoários encontrados foram: mixosporídeos, Monogenea, copepoditos e adultos de L. cyprinacea. O Argulus sp. foi observado somente em um dos "pesque-pague" em que a água de abastecimento dos viveiros provém de mina, dentro da propriedade. TOMEC et al. (1995) relataram que em condições de cultivo a baixa qualidade da água e das condições sanitárias favorecem a invasão por parasitos do gênero Argulus. Entretanto, outros fatores devem ser considerados, pois BUCHMANN et al. (1995) descreveram infestação por Argulus foliaceus somente em uma de várias truticulturas, cujo tanques recebiam água de um lago natural, a provável fonte de larvas do parasito. Assim, nesta observação o crustáceo deve ter sido introduzido na criação quando houve a introdução de peixes nos viveiros.

No "pesque-pague" 2RB a maior carga média por helmintos monogenóides ocorreu em P. mesopotamicus e L. macrocephalus.

A análise dos parâmetros físico-químicos da água demonstrou similaridade entre ambos "pesque-pague", exceto no que se refere à condutividade elétrica que 


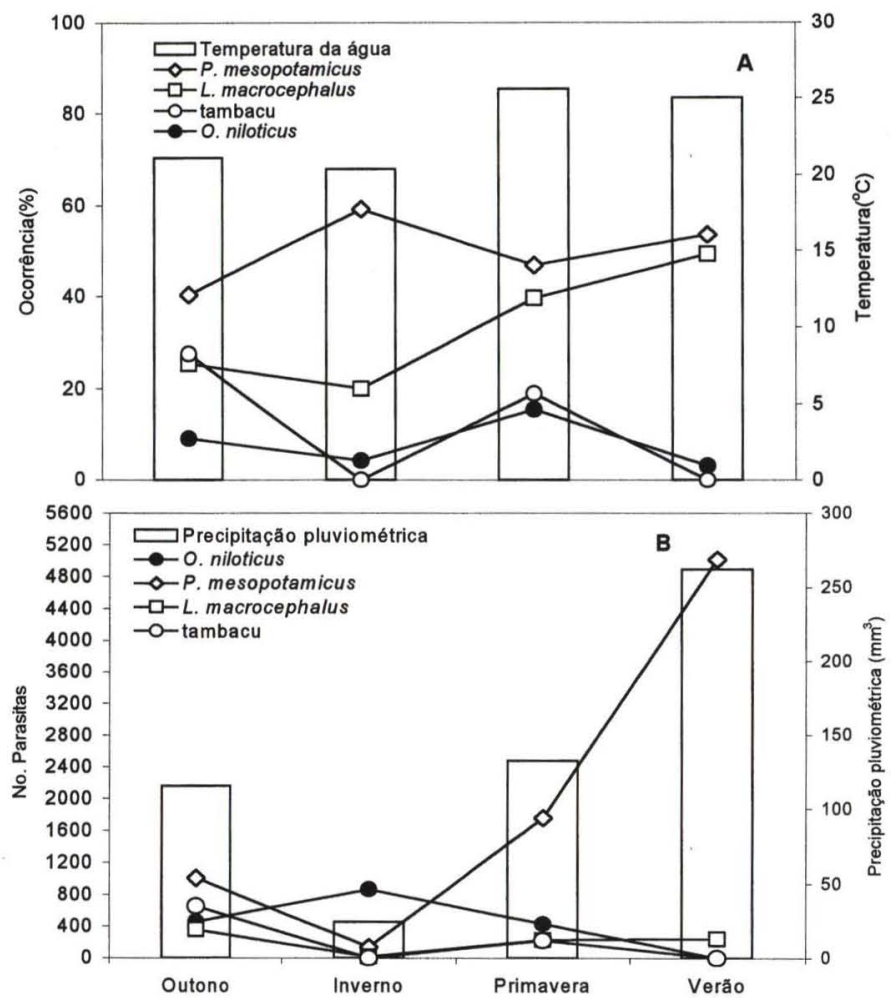

Fig. 2. Variação sazonal da temperatura da água, ocorrência (A), precipitação pluviométrica e carga parasitária média de Monogenea (B) em peixes examinados no periodo de abril de 1997 a março de 1999, em "pesque-pagues" do município de Franca, São Paulo. ( $\left.{ }^{\star}\right)$ Diferença significativa $(P<0,05)$ na ocorrência entre as estações.

apresentou maior oscilação no "pesque-pague" 2RB. De acordo com literatura infecções elevadas por monogenóides são fortes indicadores de precárias condições sanitárias e da qualidade da água (THATCHER \& BRITES-NETO 1994; NOGA 1996). As altas taxas de densidade populacional e de alimentação (FrANCIS-FLOYD \& NoGA 1994) podem levar ao aumento na concentração de amônia, nitrito e de matéria orgânica, reduzindo a quantidade de oxigênio dissolvido (THATCHER \& BRITES-NETO 1994; Noga 1996). Por isso, é de fundamental importância o conhecimento da qualidade da água na prevenção de surtos epizoóticos.

No presente trabalho, $29,3 \%$ dos espécimes examinados estavam parasitados por monogenóides, $9,7 \%$ por crustáceos e 1,6\% por mixosporídeos. Monogenea, $P$. pillulare e Trichodina sp. foram os parasitos mais freqüentemente observados. Esses percentuais foram superiores aos descritos por BÉKESI (1992), para monogenóides $(18,6 \%)$ em pisciculturas do nordeste brasileiro, mas similares para crustáceos $(11,6 \%)$ e mixosporídeos (1,6\%). MARTINS \& ROMERO (1996) estudando a fauna metazoária em pisciculturas dos Estados de São Paulo e Paraná encontraram 25,8\% de infecção por monogenóides, $14,7 \%$ por crustáceos e $8,0 \%$ por mixosporídeos. 


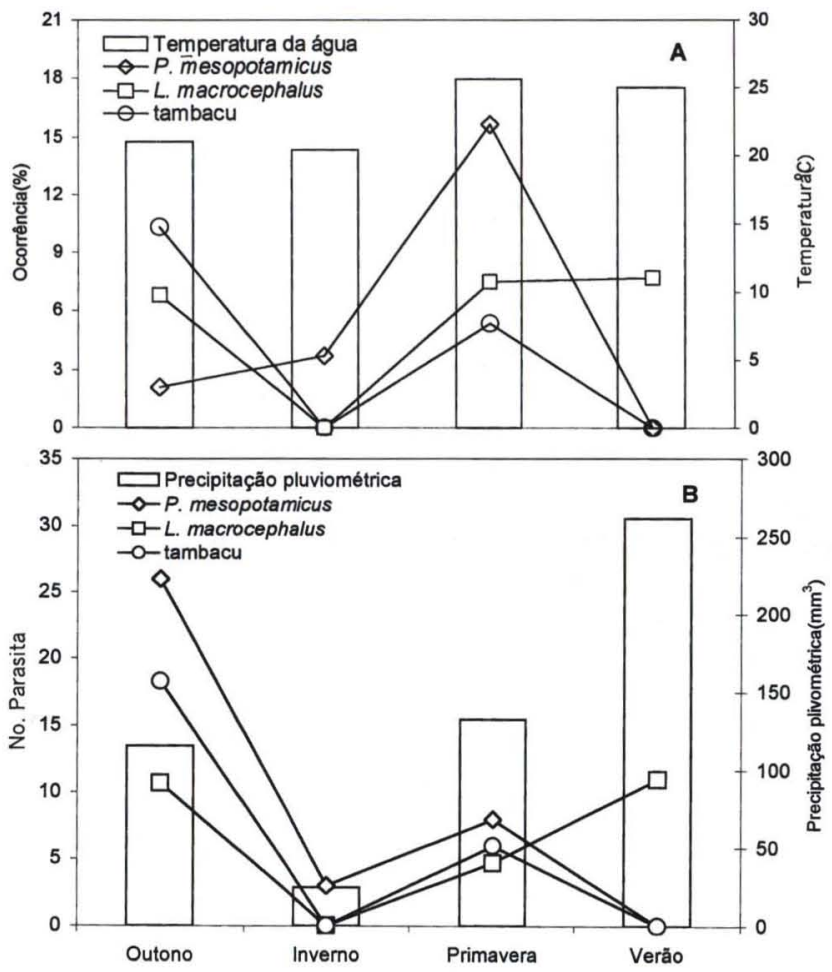

Fig. 3. Variação sazonal da temperatura da água, ocorrência (A), precipitação pluviométrica e carga parasitária média de copepoditos de $L$. cyprinacea $(B)$ em peixes examinados no período de abril de 1997 a março de 1999, em "pesque-pagues" do municipio de Franca, São Paulo. $\left(^{*}\right)$ Diferença significativa $(P<0,05)$ na ocorrência entre as estações.

Os metazoários mais freqüentes em diversas espécies de peixes necropsiados no CAUNESP, foram Monogenea e Crustacea como L. cyprinacea. Resultados similares foram descritos por TAVARES-DiAS et al. (1999), em $P$. mesopotamicus e $L$. macrocephalus cultivados em Franca.

Em truticulturas da Dinamarca, as enfermidades causadas por monogenóides, digenéticos e mixosporídeos são freqüentes (BUCHMANN \& BRESCIANI 1997). Em enguia-européia Anguilla anguilla Linnaeus, 1758 (Anguillidae), o elevado parasitismo por monogenóides foi atribuído aos hábitos gregários desse hospedeiro, à alta densidade populacional e ao tipo de manejo alimentar praticado (CRUZ-SILVA et al. 1990/1991).

No presente trabalho, somente o $P$. mesopotamicus foi susceptível à infecção por mixosporídeos (M. colossomatis e H. piaractus), porém foi baixa a ocorrência desses parasitos. Resultados similares foram descritos por BÉKÉSI (1992), nesse mesmo hospedeiro. Em contrapartida, elevada ocorrência de mixosporídeos já foi observada nas brânquias de $P$. mesopotamicus (FIGUEIRA \& CECCARELli 1991; EIRAS et al. 1995; MARTINS et al. 1997; EIRAS et al. 1998; MARTINS et al. 1999a), 

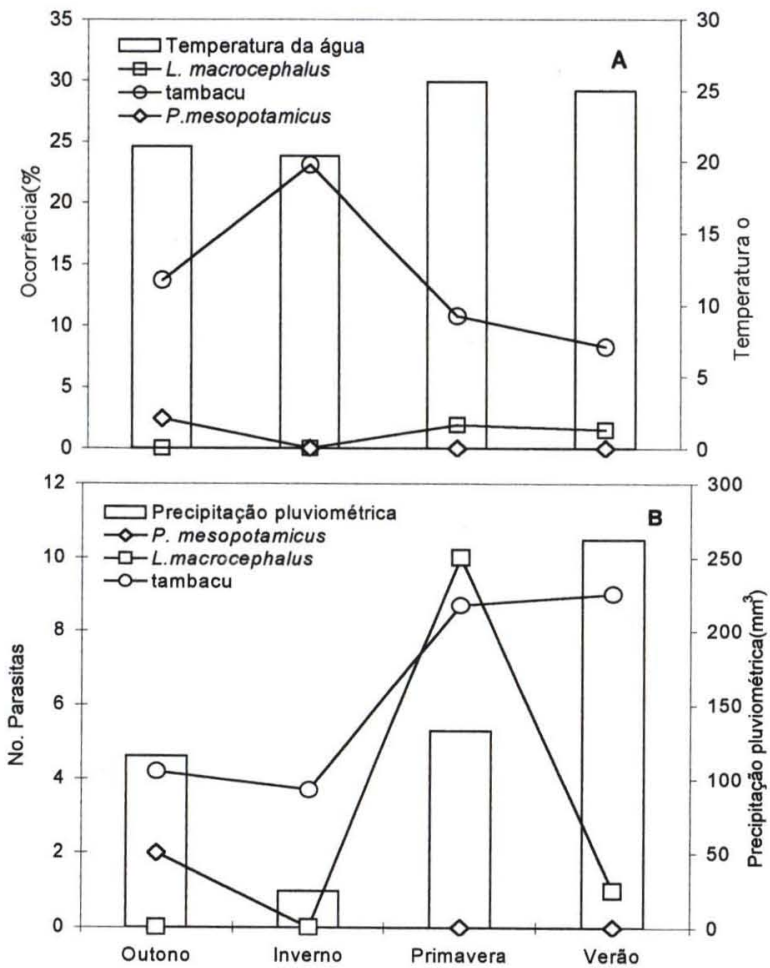

Fig. 4. Variação sazonal da temperatura da água, ocorrência $(A)$, precipitação pluviométrica e carga parasitária média de $L$. cyprinacea $(B)$ em peixes examinados no período de abril de 1997 a março de 1999, "pesque-pagues" do município de Franca, São Paulo. (*) Diferença significativa $(P<0,05)$ na ocorrência entre as estações.

tambacu (Martins et al. 1999a), perca amarela Perca flavescens Mitchill, 1814 (Percidae) (MuzZall 1995) e C. carpio (FIGUeIRA \& CECCARELli 1991; YoKoyAMA et al. 1996), criados em cultivo intensivo. Na Índia, dentre várias espécies cultivadas, apenas o bagre Rita rita Hamilton, 1822 (Bagridae) sofre infecção por tais metazoários (SINGHAL et al. 1986a).

As doenças e parasitos de peixes cultivados, podem originar-se de peixes e outros animais selvagens associados aos corpos de água. As aves piscívoras atraídas para os tanques de cultivo podem transmitir infecções de um tanque para outro (MUZZALL 1995), principalmente por esporos de mixosporídeos (THATCHER 1991). Os estudos de MARTINS et al. (1998), demonstraram que a infecção por M. colossomatis em tambacus e Astyanax bimaculatus Linnaeus, 1758 (Characidae) manifestouse após o repovoamento da represa de engorda. A enfermidade foi favorecida pelo transporte inadequado de alevinos, pela superpopulação do tanque e pelo sistema de abastecimento de água, oriundo de córrego onde havia lambaris infectados. Os lambaris foram considerados como a fonte de infecção uma vez que na propriedade de origem dos alevinos a enfermidade não havia ocorrência do parasito. Nos viveiros 
de Franca, apenas em um dos "pesque-pague" a água de abastecimento poderia ser um dos fatores responsáveis pela infecção por mixosporídeos em pacus. A água, além de passar por diversas propriedades, é repleta de lambaris, os quais podem ser reservatórios naturais desses parasitos (MARTINS et al. 1998).

Todos os teleóstos examinados neste trabalho mostraram-se susceptíveis a infecção por helmintos monogenóides, em particular o L. macrocephalus, o $P$. mesopotamicus, $C$. carpio e a $T$. rendalli. Porém, as maiores ocorrência e carga por $A$. penilabiatus ocorreram em $P$. mesopotamicus, enquanto a tilápia-do-Nilo apresentouse pouco infectada. Observações semelhantes realizadas em $P$. mesopotamicus e $C$. carpio foram descritas por vários autores no Brasil e em outros países (BÉKÉsI 1992; EIRAS et al. 1995; MARTINS \& ROMERO 1996; SINGHAL et al. 1986a; POJMANSKA \& CHABROS 1993). Entretanto, a carga parasitária descrita por POJMANSKA \& CHABROS (1993), em C. carpio é bastante inferior à verificada nesta avaliação.

Dactilogirídeos do gêneros Demidospermus e Scleroductus ocorreram em 100\% dos espécimes de Pimelodus maculatus Lacépede, 1803 (Pimelodidae) do Rio de la Plata (Argentina), com intensidade média variando entre 9,4 e 82,9 parasitos (GUTIÉRREZ \& MARTORELli 1999). Em A. anguilla de duas localidades do Rio Rhine (Alemanha) a ocorrência de Pseudodactylogyrus sp. variou de 45,0 a $45,9 \%$ (SURES et al. 1999). Na Dinamarca, A. anguilla de lago eutrofizado infectadas por duas espécies de Pseudodactylogyrus evidenciaram ocorrência de 3,3\% a $66,7 \%$, com média de 1,5 a 10,0 parasitos por hospedeiro (KoIE 1988). Enquanto nesse mesmo hospedeiro de tanques de cultivo, a ocorrência foi de $45, \%$, com média de 1,0 parasito por peixe (BUCHMANN 1989). De acordo com os estudos de CHUBB 1977), tais disparidades estão relacionadas ao fato de que é grande a variação no padrão de ocorrência dos monogenóides, até entre membros do mesmo gênero. Algumas espécies demonstram alterações mínimas de incidência e intensidade de ocorrência durante o ano. Outras evidenciam alterações mínimas de ocorrência, mas alterações consideráveis de intensidade. Enquanto outras possuem grande alteração em ambos, muitas vezes com período de ausência no hospedeiro. O padrão de ocorrência e intensidade de infecção podem estar relacionados em maior ou menor grau aos efeitos de fatores abióticos como a concentração de oxigênio e temperatura da água ou a fatores bióticos como a espécie hospedeira, seu comportamento, migrações, imunidade ou à interação de todos esses fatores.

Entretanto, o estado fisiológico do hospedeiro no momento da infecção também pode influenciar na habilidade de resistência a doenças (PICKERING \& POTTINGER 1985). JANSEN \& BAKKE (1995) concluíram que em S. trutta privadas de alimentação, a persistência do monogenóide Gyrodactylus salaris Malmberg, 1957 (Gyrodactylidae) foi maior quando comparado aos peixes alimentados.

Copepoditos de L. cyprinacea foram encontrados nas brânquias de $P$. mesopotamicus, tambacu e L. macrocephalus. Mas a maior ocorrência foi em $L$. macrocephalus. As formas adultas de L. cyprinacea foram observadas apenas em $P$. mesopotamicus e no tambacu, sendo esse último hospedeiro o portador da maior carga média de parasitos. As tilápias e carpas não estavam parasitadas. Estudos similares relatam alta ocorrência de $L$. cyprinacea em $P$. mesopotamicus, tambacu (MARTINS \& ROMERO 1996; VILEM et al. 1998), L. macrocephalus (ALEXANDRINO 
et al. 1999), em carpas comum C. carpio e capim Ctenopharyngodon idella Valenciennes, 1844 (Cyprinidae) (ForTES et al. 1998). Contrariamente, outros autores relatam a ocorrência desse parasito em C. idella, Brycon lundii Reinhardt, 1874 (Characidae), lambaris e C. carpio mas não em P. mesopotamicus, C. macropomum e tambacu, embora não tenha sido mencionado se os animais eram conviventes no mesmo ambiente (CECCARELLi et al. 1990). Peixes que possuem escamas menores como o $P$. mesopotamicus são menos susceptíveis a lerneose enquanto espécies com escamas maiores como $C$. idella, o B. cephalus e C. carpio teriam maior susceptibilidade (CECCARELLi 1988). Os dados deste trabalho indicam o contrário.

Nos peixes examinados em Franca, o parasitismo por Argulus sp. foi baixo, ocorrendo somente em L. macrocephalus e em P. mesopotamicus coincidindo com as observações de MARTins \& ROMERO (1996). Entretanto, em C. carpio de pisciculturas do nordeste do Brasil, há diagnóstico de elevado percentual de infestação (BÉKÉSI 1992). Na Índia, o Argulus indicus Weber (Argulidae) é responsabilizado por altas taxas de mortalidade em ciprinídeos como Catla catla Hamilton, 1822 (Cyprinidae), Labeo rohita Hamilton, 1822 (Cyprinidae), Cirrhinus mrigala Hamilton, 1822 (Cyprinidae), C. idella e Hypophthalmichthys molitrix Valenciennes, 1844 (Cyprinidae) criados em cultivo intensivo (Singhal et al. 1986b). Infestação elevada por parasitos do gênero Argulus é mais comum em peixes jovens, mantidos em tanques de cultivo com alta densidade populacional (CAMPBELL 1971) e baixas qualidade de água e de condições sanitárias, além de alta temperatura (Singhal et al. 1986a; TOMEC et al. 1995).

Em P. mesopotamicus, O. niloticus, L. macrocephalus e tambacu a ocorrência de helmintos monogenóides não sofreu influência sazonal significativa, mas houve infecção durante todo o ano. Entretanto, a carga média de Monogenea em $P$. mesopotamicus (A. penilabiatus) e em L. macrocephalus foi menor nos meses de inverno e aumentou na primavera e verão acompanhando a variação da temperatura. No híbrido tambacu, a infecção ocorreu nos meses de outono e primavera mas não no inverno e verão. Esses resultados corroboram parcialmente as observações de MEYER (1969), onde a ocorrência desses helmintos é observada mais freqüentemente nos meses de primavera e verão. Em tainha Mugil liza Valenciennes, 1836 (Mugillidae), da costa do Rio de la Plata, a maior taxa de infecção por Monogenea do gênero Gyrodactylus ocorreu na primavera e verão (CARNEVIA et al. 1988). Entretanto, em C. carpio, cultivadas na India, a maior ocorrência de parasitos desse mesmo gênero foi nos meses de primavera e inverno (SINGHAL et al. 1986a). CHAPPELL (1969) verificou que no hospedeiro esgana-gata Gasterosteus aculeatus Linnaeus, 1776 (Gasterosteidae) o máximo de desenvolvimento embrionário do Gyrodactylus rarus Wegener, 1910 (Gyrodactylidae), ocorreu nos meses de primavera. Todavia devem ser levadas em conta as diferenças climáticas das diferentes regiões onde foram realizadas tais observações.

O aumento da intensidade de infecção por Gyrodactylus sp., em Oncorhynchus mykiss Walbaum, 1792 (Salmonidae) apresenta relações com as baixas temperaturas (BUCHMANN \& BRESCIANI 1997), enquanto que resultados contrários foram observados em Salmo trutta Linnaeus, 1758 (Salmonidae) portadores 
de maiores carga parasitária e ocorrência de Gyrodactylus derjavini Mikailov, 1975 (Gyrodactylidae) na primavera e verão, quando a temperatura é mais alta (Mo 1997). Por outro lado, em Salmo salar Linnaeus, 1758 (Salmonidae), a ocorrência de G. salaris é elevada durante todo ano, exceto no inverno. O número de parasitos é maior no verão e diminui nos meses de inverno e primavera (APPLEBY \& Mo 1997).

De acordo com EIRAS (1994) a maior parte das espécies de Monogenea tem padrão de infecção anual bem definido. Há incremento da ocorrência e do número de parasitos no verão, redução nos meses frios e é mínimo na primavera. Outras afastam-se nitidamente desse comportamento, apresentando valores elevados durante todo o ano. Nesse caso os parasitos tem características especiais do ciclo vital, que permitem processos de infecção reincidentes e contínuos. $O$ fator temperatura é também importante para o ciclo biológico dos helmintos monogenóides como ilustra o fato de que 0 Dactylogyrus vastator Nybelin, 1924 (Dactylogyridae) sobrevive de 20 a 25 dias em temperaturas de verão e por seis a sete meses no inverno.

A infecção por copepoditos e adultos de L. cyprinacea não demonstrou alteração sazonal significativa. Porém, em tambacus não houve parasitismo por copepoditos no inverno e as infestações por adultos de $L$. cyprinacea foram contínuas, com o máximo de parasitos na primavera e verão. Em brânquias do hospedeiro $P$. mesopotamicus não houve infecção por copepoditos nos meses de verão, mas o maior número de formas imaturas foi observado no outono, coincidindo com o máximo de parasitos na forma adulta. Em L. macrocephalus não foi observada a presença de copepoditos no período de inverno e de $L$. cyprinacea no outono e inverno e a maior carga parasitária com adultos ocorreu na primavera.

Em Micropterus salmoides Lacépede, 1802 (Centrarchidae) a infestação por tais crustáceos aumenta juntamente com a temperatura (TIMMONS \& HEMSTREET 1980). Em H. molitrix a presença de L. cyprinacea é contínua sendo que as menores infestações ocorrem nos meses de baixas temperaturas (SINGHAL et al. 1996a), quando os parasitos não completam seu ciclo de vida (NoGA 1996). Por isso, as infestações por adultos de L. cyprinacea são mais freqüentes na primavera e verão enquanto no outono e inverno geralmente são observados na superfície corporal dos peixes ou nas brânquias as formas imaturas, os copepoditos (MARTINS 1997). A carga parasitária apresenta relações com o tamanho do hospedeiro, já que os peixes mais velhos, e portanto mais volumosos, têm número maior de parasitos (EIRAS 1994) e os muito pequenos não evidenciam infestação parasitária (TIMMONS \& HEMSTREET 1980).

\section{CONCLUSÕES}

Por ordem decrescente o grau de suscetibilidade dos hospedeiros aos parasitos metazoários foi $P$. mesopotamicus, L. macrocephalus, tambacu, C. carpio, $B$. cephalus, $T$. rendalli e $O$. niloticus. Não ocorreu interferência sazonal estatisticamente significativa em nenhuma das espécies de peixe para monogenóides, copepoditos e adultos de L. cyprinacea. Todavia, o significado biológico das variações sazonais não devem ser esquecidos. 


\section{REFERÊNCIAS BIBLIOGRÁFICAS}

Alexandrino, A.C.; L.M.S. Ayrosa; M.P.M. Okumura; E. Liuson; A.O.S. Pauli; M.B. Rosa \& T.M.P.A. CarvalhaEs. 1999. Infestação por Lernaea cyprinacea Linnaeus, 1758 (Crustacea, Copepoda) em cultivo semi-intensivo de piauçu (Leporinus macrocephalus Valenciennes, 1877) na região do Vale do Paranapanema - Relato de Caso. A Hora Veterinária 19 (109): 65-67.

AppleBy, C. \& T.A. Mo. 1997. Populations dynamics of Gyrodactylus derjavini (Monogenea) infecting Atlantic salmon, Salmo salar, parr in the River Batnfjorselva, Norway. Jour. Parasitol. 83 (6): 23-30.

BÉKÉSI, L. 1992. Evaluation of data on ichthyopathological analyses in the Brazilian Northeast. Ciência Cultura 44 (6): 400-403.

BUCHMANN, K. 1989. Relationship between host size of Anguilla anguilla and the infections level of the monogeneas Pseudodactylogyrus spp. Jour. Fish Biol. 35: 599-601.

Buchmann, K.; A. Uldal \& H.C.K. Lyholt. 1995. Parasite infections in danish trout farms. Acta Vet. Scand. 36: 283-298.

BuchmanN, K. \& J. BRESCIANI.1997. Parasitic infections in pond-reared rainbow trout Oncorhynchus mykiss in Denmark. Dis. Aquat. Organ. 25: 125-138.

CAmpBel., A.D. 1971. The occorrence of Argulus (Crustacea: Branchiura) in Scotland. Jour. Fish Biol. 3: $145-146$.

Carnevia, D.; R. Mazzoni; O. Areosa; T. Eastman \& D. Lorenzo. 1988. Variaciones estacionales de diversas parasitosis em la lisa Mugil liza - Val. 1836 del Rio de La Plata. An. VI Simp. Latinoamer. e V Simp. Brasil. Aqüicul., Florianópolis, p. 299-304.

CeCCarelli, P.S. 1988. Susceptibilidade à infestação de Lernaea Copepoda, Lernaeidae Linnaeus, em diferentes espécies de peixes cultivados no CEPTA e testes de infestação do pacu Piaractus mesopotamicus em laboratório. Bol. Téc. CEPTA 1 (2): 31-35.

Ceccarelli P.S.; L.B. Figueir^; C.L.B. Ferr^z lim^ \& C.A. Oliveir^. 1990. Observações sobre a ocorrência de parasitos no CEPTA entre 1983 e 1990. Bol. Téc. CEPTA 3: 43-55.

Chammas, M. 1997. Panorama da aqüicultura brasileira - A região sul. I An. Workshop Intern. Aqüicul., São Paulo, p. 56-61.

Chappell, L.H. 1969. The parasites of the three-spined stickleback Gasterosteus aculeatus L. from a Yorkshire pond. I. Seasonal variation of parasite fauna. Jour. Fish Biol 1: 137-152.

ChÁvez-MANCILLA, E.L. 1985. Análisis patológico de las alteraciones producidas por ectoparásitos en reproductores de tilapia Sarotherodon hornorum (Trewavas) y Oreochromis mossambicus (Peters).

Rev. Latinoamer. Acui 25: 24-28.

Chuвb, J.C. 1977. Seasonal occurrence of helminths in freshwater fishes. Part I. Monogenea. Parasitology 15: 133-198.

Cruz-Silva, M.P.; M. Carval.ho-Varell..A; S.P. Carvalho \& S. Grazina-Freitas. 1990/1991. Parasitas e parasitoses dos animais aquáticos em estuarino e maricultura em Portugal. Resultados preliminares. An. Fac. Medic. Vet. Lisboa 27/28: 223-236.

Delariva, R.L. \& A.A. Agostinho. 1999. Introdução de espécies: Uma síntese comentada. Acta Scientiarum 21 (2): 255-262.

DiAs, E.R.A. 1998. Piscicultura no Estado de São Paulo. Bol. Inform. Ass. Patol. Organ. Aquát. 15: $8-10$.

EIRAS, J.C. 1994. Elementos de Ictioparasitologia. Porto, Fund. Eng. Antônio de Almeida, 339p.

Eiras, J.C.; M.J.T. Ranzani-Paiva; C.M. Ishikawa; A.C. Alexandrino \& A.C. Eiras.1995. Ectoparasites of semi-intensively farmed tropical freshwater fish Piaractus mesopotamicus, Prochilodus lineatus and Colossoma macropomum in Brazil. Bull. Eur. Ass. Fish Pathol 15 (5): 148-151.

Eiras, J.C.; G.C. Pavanelli; J.A. Sousa; R.M. Takemoto \& M.J.T. Ranzani-Paiva. 1998. Ocorrência de agentes com potencial patogênico em peixes de dois pesque-pague e uma piscicultura do norte do Estado do Paraná. An. Aqüicult. Brasil 98 2: 697-701.

Figueira, L.B. \& P.S. CFCCAREI.L.I. 1991. Observações sobre a presença de ectoparasitas em pisciculturas 
tropicais de interior (CEPTA e região). Bol. Téc. CEPTA 4 (1): 57-65.

Fortes, E.; R.P. HoFmann \& J. SCARIOT. 1998. Lernaea cyprinacea Linnaeus, 1758 (Crustacea, Copepoda) parasitando peixes de água doce da Grande Porto Alegre, RS, Brasil. Rev. Brasil. Med. Vet. 20 (2): 64-65.

Francis-Floyd, R. \& E.J. Noga. 1994. Medical management of channel catfis. Part I. Types of skin and gill pathogens. Compend. Contin. Educ. Pract. Vet. 16 (6): 808-814.

Grosman, F. 1999. Estrutura da comunidade de peixes da represa "Lago del Fuente", Tandil, Argentina. Acta Scientiarum 21 (2): 267-275.

GutiéRREZ, P.A. \& S.R. MARTORELLI. 1999. The struture of the monogenean community on the gills of Pimelodus maculatus in Rio de la Plata (Argentina). Parasitology 119: 177-182.

HEDRICK, R.P. 1998. Relationships of the host, patogen, and environment. Implications for deseases of cultured and wild fish populations. Jour. Aquat. Anin. Health 10: 107-111.

JANSEN, P.A. \& T.A. BAKKE. 1995. Susceptibility of brown trout to Gyrodactylus salaris (Monogenea) under experimental conditions. Jour. Fish Biol. 46 (3): 415-422.

KENNEDY, C.R. 1993. Introductions, spread and colonization of new localities by fish helminth and crustacean parasites in the British Isles: a perspective and appraisal. Jour. Fish Biol. 43: 287-301.

KolE, M. 1988. Parasites in eels, Anguilla anguilla (L), from eutrophic Lake Esrum (Denmark). Acta Parasitol. Polonica 3 (2): 89-100.

MARTINS, M.L. 1997. Principais doenças nos peixes brasileiros. I An. Workshop Intern. Aqüicul., São Paulo, p. 90-95.

. 1998. Doenças infecciosas e parasitárias de peixes. Jaboticabal, FUNEP, $2^{\mathrm{a}}$ ed., $65 \mathrm{p}$.

Martins, M.L. \& N.G. Romero. 1996. Efectos del parasitismo sobre el tejido branquial en peces cultivados: estudio parasitologico e histopatologico. Revta bras. Zool. 13 (2): 489-500.

Martins, M.L.; V.N. Souza; F.R. Moraes; J.R.E. Moraes; A.J. Costa \& U.F. Rocha. 1997. Pathology and behavioral effects associated with Henneguya sp. (Myxozoa: Myxobolidae) infections of captive pacu Piaractus mesopotamicus in Brazil. Jour. World Aquac. Soc. 28 (3): 297-300.

MARTINS, M.L.; V.N. SouZA \& F.R. Moraes. 1998. Infecção por Myxobolus colossomatis (Myxozoa: Myxobolidae) em alevinos de "tambacus" oriundos de piscicultura comercial. "Lambaris" como possivel fonte de infecção. Ars Veterinaria 14 (3): 324-330.

Martins, M.L.; V.N. SouzA; J.R.E. Moraes, F.R Moraes \& A.J. CostA. 1999a. Comparative evaluation of the susceptibility of cultivated fishes to the natural infection with myxosporean parasites and tissue changes in the host. Rev. Brasil. Biol. 59 (2): 263-269.

Martins, M.L.; V.N. Souza; J.R.E. Moraes \& F.R Moraes. 1999b. Gill infection of Leporinus macrocephalus Garavello \& Britski, 1988 (Osteichthyes: Anostomidae) by Henneguya leporinicola n. sp. (Myxozoa: Myxobolidae). Description, histopathology and treatment. Rev. Brasil. Biol. 59 (3): 527-534.

MAYER, F.P. 1969. Seasonal fluctuations in the incidence of disease on fish farms. Dis. Fish Shelf. 52: 21-29.

Mo, T.A. 1997. Seasonal occurrence of Gyrodactylus derjavini (Monogenea) on brown trout, Salmo trutta, and Atlantic salmon, S. salar, in the Sanddvikselva River, Norway. Jour. Parasitol. 83 (6): 1025-1029.

Muzzall, P.M. 1995. Parasites of pond-reared yellow perch from Michigan. Prog. Fish-Culturist 57: 168-172.

NogA, E.J. 1996. Fish Disease. Diagnosis and Treatment. St. Louis, Mosby-Year Book Inc., 367p.

ORSI, M.L. \& A.A. AgostinHo. 1999. Introdução de espécies de peixes por escapes acidentais de tanques de cultivo em rios da Bacia do Rio Paraná, Brasil. Revta bras. Zool. 16 (2): 557-560.

Pavanelli, G.C.; J.C. EirAs \& R.M. TAKEMOTO.1998. Doenças de peixes: profilaxia, diagnóstico e tratamento. Maringá, Ed. EDUEM, 259p.

PiCkering, A.D. \& T.G. Pottinger. 1985. Cortisol can increase the susceptibility of brown trout, Salmo trutta L., to disease without reducing the white blood cell count. Jour. Fish Biol. 27: 611-619.

Pojmanska, T. \& M. Chabros. 1993. Parasites of common carp and three introduced cyprinid fish in 
pond culture. Acta Parasitol. 38 (3): 101-108.

Ranzant-Paiva, M.J.; A.L. Vieira \& C.M. IshiKawa.1989. Análise dos constituintes do plasma sangüíneo da carpa, Cyprinus carpio, infestada por Argulus sp. Bol. Inst. Pesca 16 (1): 117-121.

RENO, P.W. 1998. Factors involved in the dissemination of desease in fish populations. Jour. Aquat. Anin. Health 10: 160-171.

Roberts,R.J. 1981. Patologia de los peces. Madrid, Mundi-Prensa, 366p.

Singhal, R.N.; S. JeET \& R.W. Davies. 1986a. The relationships between changes in selected physico-chemical properties of water and the occurrence of fish parasites in Haryana, India. Trop. Ecol. 27: 1-9. 165-171.

SurES, B.; K. KNOPF; J. WÜRTZ. \& J. HiRT. 1999. Richness and diversity of parasite communities in European eels Anguilla anguilla of River Rhine, Germany, with special reference to helmintes parasites. Parasitology 119: 323-330.

Tavares-Dias, M; M.L. Martins \& F.R. Moraes. 2001. Fauna parasitária de peixes oriundos de "pesque-pague" do município de Franca, São Paulo, Brasil. I. Protozoários. Revta bras. Zool. 18 (Supl. 1): 67-79.

Tavares-Dias, M; S.H.C. Schalch; M.L. Martins; E.D. Silva; F.R. Moraes \& D. Perecin. 1999. Hematologia de teleósteos brasileiros com infecção parasitária. I. Variáveis do Leporinus macrocephalus Garavello \& Britski, 1988 (Anostomidae) e Piaractus mesopotamicus Holmberg, 1887 (Characidae). Acta Scientiarum 21: 337-342.

ThatCher, V.E. 1991. Amazon fish parasites. Amazoniana 11 (3/4): 263-572.

ThATChER, V.E. \& J. Brites-Neto. 1994. Diagnósitco, prevenção e tratamento das enfermidades de peixes neotropicais de água doce. Rev. Brasil. Med. Vet. 16 (3): 111-128.

Timmons, T.J. \& W.G. Hemstreet. 1980. Prevalence rate of Lernaea cyprinacea L. (Copepoda: Lernaeidae) on young-of-the-year largemouth bass, Micropterus salmoides (Lacépede), in West Point Reservoir, Alabama-Georgia, U.S.A. Jour. Fish Dis. 3: 529-530.

Tomec, M.; M. Hacmanjek; Z. TeskeredziC; E. Teskeredzic \& R. Coz-Rakovac. 1995. Kvaliteta vode i ektoparazitarne bolesti ciprinidnih riba. Ribarstvo 53 (4): 129-139.

Vilem, R.; C.R. Del-Carratore \& J.H. Machado.1998. Eficácia do tratamento terapêutico com demetil-parathion e ascículas de pinus (Pinnus elliot) em peixes acometidos por lerneoses (Lernaea sp). An. Aqüicult. Brasil 98 2: 689-695.

YokoYama, H.; T. Danjo; K. Ogawa; T. Arima \& H. WaKabayashi. 1996. Hemorrhagic anemia of carp associated with spore discharge of Myxobolus artus (Myxozoa: Myxosporea). Fish Pathol. 31 (1): 19-23.

Recebido em 13.VII.2000; aceito em 19.VI.2001. 